\title{
X-Ray Diffuse Scattering Measurements of Nucleation Dynamics at Femtosecond Resolution
}

\author{
A. M. Lindenberg, ${ }^{1,2,3}$ S. Engemann, ${ }^{1,3}$ K. J. Gaffney, ${ }^{1,3}$ K. Sokolowski-Tinten, ${ }^{4}$ J. Larsson, ${ }^{5,1}$ P. B. Hillyard, ${ }^{1,6}$ \\ D. A. Reis, ${ }^{7,1}$ D. M. Fritz,${ }^{1,7}$ J. Arthur, ${ }^{3}$ R. A. Akre, ${ }^{8}$ M. J. George, ${ }^{3}$ A. Deb,${ }^{1,3}$ P. H. Bucksbaum,,${ }^{1,3}$ J. Hajdu, ${ }^{9,1}$ \\ D. A. Meyer, ${ }^{10,1}$ M. Nicoul, ${ }^{4}$ C. Blome, ${ }^{11}$ Th. Tschentscher, ${ }^{11}$ A. L. Cavalieri, ${ }^{7,12}$ R. W. Falcone, ${ }^{13}$ S. H. Lee, ${ }^{14}$ R. Pahl, ${ }^{15}$ \\ J. Rudati, ${ }^{16}$ P. H. Fuoss, ${ }^{17}$ A. J. Nelson, ${ }^{18}$ P. Krejcik, ${ }^{8}$ D. P. Siddons, ${ }^{19}$ P. Lorazo, ${ }^{20}$ and J. B. Hastings ${ }^{3}$ \\ ${ }^{1}$ PULSE Center, Stanford Linear Accelerator Center, Menlo Park, California 94025, USA \\ ${ }^{2}$ Stanford University, Department of Materials Science and Engineering, Stanford, California 94305, USA \\ ${ }^{3}$ Stanford Synchrotron Radiation Laboratory/SLAC, Menlo Park, California 94025, USA* \\ ${ }^{4}$ Institut für Experimentelle Physik, Universität Duisberg-Essen, D-45117 Essen, Germany \\ ${ }^{5}$ Department of Physics, Lund Institute of Technology, P.O. Box 118, S-22100 Lund, Sweden \\ ${ }^{6}$ Department of Chemistry, Stanford University, Stanford, California 94305, USA \\ ${ }^{7}$ FOCUS Center, Department of Physics and Applied Physics Program, University of Michigan, Ann Arbor, Michigan 48109, USA \\ ${ }^{8}$ Stanford Linear Accelerator Center, Menlo Park, California, 94025, USA \\ ${ }^{9}$ Department of Cell and Molecular Biology, Biomedical Centre, Uppsala University, SE-75124 Uppsala, Sweden \\ ${ }^{10}$ Stanford University, Department of Chemistry, Stanford, California 94305, USA \\ ${ }^{11}$ Deutsches Elektronen-Synchrotron DESY, Notkestrasse 85, 22607 Hamburg, Germany \\ ${ }^{12}$ Max-Planck-Institute of Quantum Optics, Hans-Kopfermann-Str. 1, D-85748, Garching, Germany \\ ${ }^{13}$ Department of Physics, University of California, Berkeley, California 94720, USA \\ ${ }^{14}$ Length/Time Metrology Group, Korea Research Institute of Standards and Science, Daejeon 305-600, Republic of Korea \\ ${ }^{15}$ Consortium for Advanced Radiation Sources, The University of Chicago, Chicago, Illinois 60637, USA \\ ${ }^{16}$ Advanced Photon Source, Argonne National Laboratory, Argonne, Illinois 60439, USA \\ ${ }^{17}$ Materials Science Division, Argonne National Laboratory, Argonne, Illinois 60439, USA \\ ${ }^{18}$ Physics Department, Lawrence Livermore National Laboratory, Livermore, California 94550, USA \\ ${ }^{19}$ National Synchrotron Light Source, Brookhaven National Laboratory, Upton, New York 11973, USA \\ ${ }^{20}$ Departement de Genie Physique, Ecole Polytechnique de Montreal, \\ C.P. 6079, Succursale Centre-Ville, Montreal (Quebec), Canada H3C 3A7
}

(Received 29 November 2007; published 31 March 2008)

\begin{abstract}
Femtosecond time-resolved small and wide angle $\mathrm{x}$-ray diffuse scattering techniques are applied to investigate the ultrafast nucleation processes that occur during the ablation process in semiconducting materials. Following intense optical excitation, a transient liquid state of high compressibility characterized by large-amplitude density fluctuations is observed and the buildup of these fluctuations is measured in real time. Small-angle scattering measurements reveal snapshots of the spontaneous nucleation of nanoscale voids within a metastable liquid and support theoretical predictions of the ablation process.
\end{abstract}

DOI: 10.1103/PhysRevLett.100.135502

PACS numbers: 79.20.Ds, 61.05.cp, 61.20.Lc, 64.60.-i

The liquid state of matter can be both supercooled below its freezing point and superheated above its boiling point. Although these are nonequilibrium states, their lifetime is longer than typical laboratory observation times and they are thus classified as metastable, stuck in a local minimum of the free energy. Despite their apparent stability, these systems exhibit localized fluctuations, corresponding to nuclei of the lower energy phase which rapidly form and dissolve, a process for which there exists a critical size for stable growth of the equilibrium phase, typically of nanoscale dimensions [1-3] (depending on the degree of superheating). When the transition does occur, it occurs rapidly, as is familiar to anyone who has observed the catastrophic boiling that suddenly occurs in superheated liquid water [4]. The development of ultrafast, atomic-scale sensitive techniques enables one to capture these transient, intermediate states, which under current laboratory methods, may spontaneously transform to lower energy stable states on inaccessible time scales. Techniques like time-resolved $\mathrm{x}$-ray or electron scattering record these intermediate states in the form of a diffuse scattering pattern whose shape reflects the local atomic-scale structure that characterizes the transforming material. As a result of the orientational isotropy in the system and the short correlation lengths, these are typically broad diffraction rings, in contrast to the well-defined diffracted beams one obtains from a crystalline system. The radially averaged diffracted intensity as a function of scattering angle (normalized by atomic scattering factors) is a direct measure of the liquid structure factor $S(Q)$, where $Q=4 \pi \sin \theta / \lambda$ is the momentum transfer, $2 \theta$ is the scattering angle, and $\lambda$ is the wavelength, which directly encodes the short-range correlations in disordered materials.

Recent experiments probing atomic-scale dynamics in disordered systems using electron diffraction techniques have focused on gas-phase photochemical reactions [5], 
the melting transition in thin-film metallic or semiconducting samples [6,7], and other interfacial phenomena [8] on ps and subps time scales. In contrast, the weak scattering cross section of $\mathrm{x}$ rays compared to electrons allows for bulk probing of condensed phase dynamics, but has so far limited x-ray scattering studies of disordered or liquid systems to 100 ps time scales [9-12]. We describe here diffuse $\mathrm{x}$-ray scattering measurements in which the structural dynamics of the liquid-state and the subsequent ablation process are directly measured with subpicosecond resolution. Measurements were performed at the Stanford linear accelerator center using the sub-picosecond pulse source, a precursor to future hard $\mathrm{x}$-ray free electron lasers [13] and a spontaneous source of $9 \mathrm{keV} \mathrm{x}$-rays with a pulse duration of $80 \mathrm{fs}$ FWHM [14]. The setup is similar to the schematic shown in reference [15]. An InSb bulk singlecrystalline (100) sample was placed so that the X-rays were incident at 0.4 degrees, without satisfying the Bragg condition for any reflection. A $50 \mathrm{fs}, 800 \mathrm{~nm}$ laser pulse at a fluence of $\sim 75 \mathrm{~mJ} / \mathrm{cm}^{2}$ was incident near Brewster's angle. In order to overcome timing fluctuations between $\mathrm{x}$ rays and laser, an electro-optic sampling technique $[16,17]$ was used to record the relative arrival time of each X-ray pulse with respect to the pump laser pulse. Under these laser excitation conditions, a nonthermal solid-liquid transition occurs on subpicosecond time scales $[15,18,19]$, followed by isochoric heating of the optically formed liquid to high temperatures and pressures. Near the ablation threshold [20], the sample then relaxes through adiabatic expansion into a region of the phase diagram lying between the binodal and spinodal curves, in the liquid-vapor coexistence regime [21,22]. Here the system is metastable, and a rapid phase-explosion-driven boiling is thought to occur, leading to ablation of the near-surface layer, with a threshold fluence of roughly $50 \mathrm{~mJ} / \mathrm{cm}^{2}$ in InSb. We have probed this process directly by recording the time-dependent scattering from the near-surface region on a charge-coupled device (CCD) camera, placed just beyond the edge of the sample.

Figure 1 shows raw snapshots of the scattering from the liquid state for a range of times before and after optical excitation, capturing the full range of dynamics from liquid formation to recrystallization. Following excitation, a broad diffuse scattering ring emerges which rapidly narrows, shifts to smaller scattering angles, and eventually dissolves as a result of material removal and recrystallization of the underlying liquid layer. Figure 2(a) shows radially-averaged lineouts of measured scattering patterns, normalized by the atomic scattering factor squared (corrected for polarization and geometric effects) to give the liquid structure factor $S(Q, t)$. Also shown are MD simulations by $\mathrm{Gu}$ et al. [23] of equilibrium liquid $\mathrm{InSb}$ at its melting temperature, corrected for our $Q$ resolution. At long times $(t=20 \mathrm{~ns})$, good agreement with the equilibrium liquid state is obtained. This equilibrium structure is observed to last for at least $100 \mathrm{~ns}$, indicating that this arises from an attached liquid surface layer and not an ablated liquid layer (which would be unstable on ns time scales and of different density than the equilibrium liquid [21]). In contrast, at short times, the liquid structure factor is significantly different than that for the equilibrium liquid. At $t=500 \mathrm{fs}$ (shorter than the time scale for transfer of energy to the lattice), the structure factor is significantly broadened compared to the equilibrium case as the local structure of the liquid or disordered state is just beginning to form. By $t=10 \mathrm{ps}$, and lasting for hundreds of ps, large deviations from the equilibrium structure factor are observed at small scattering angles.

The time scale on which the diffuse scattering increases is displayed in Fig. 3, showing the integrated diffuse scattering as a function of time for both the high $Q$ (1.0-3.0 $\AA^{-1}$, near the peak of the liquid structure factor) and low $Q\left(0.4-1.6 \AA^{-1}\right.$, small angle) regions. Also shown in Fig. 3 is the time-dependence of the (111) Bragg scat-
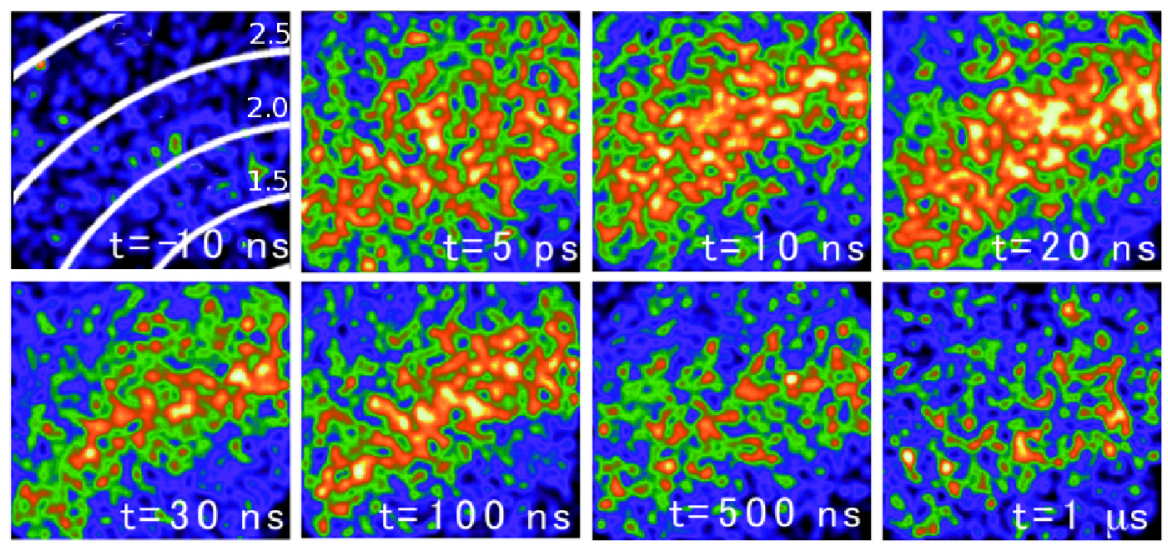

FIG. 1 (color online). Raw snapshots of the liquid structure factor on time scales from picoseconds to microseconds, as recorded on a CCD camera placed just behind the sample. The top left image shows lines of constant $Q$, corresponding to collection of a fraction of the diffuse scattering ring. 

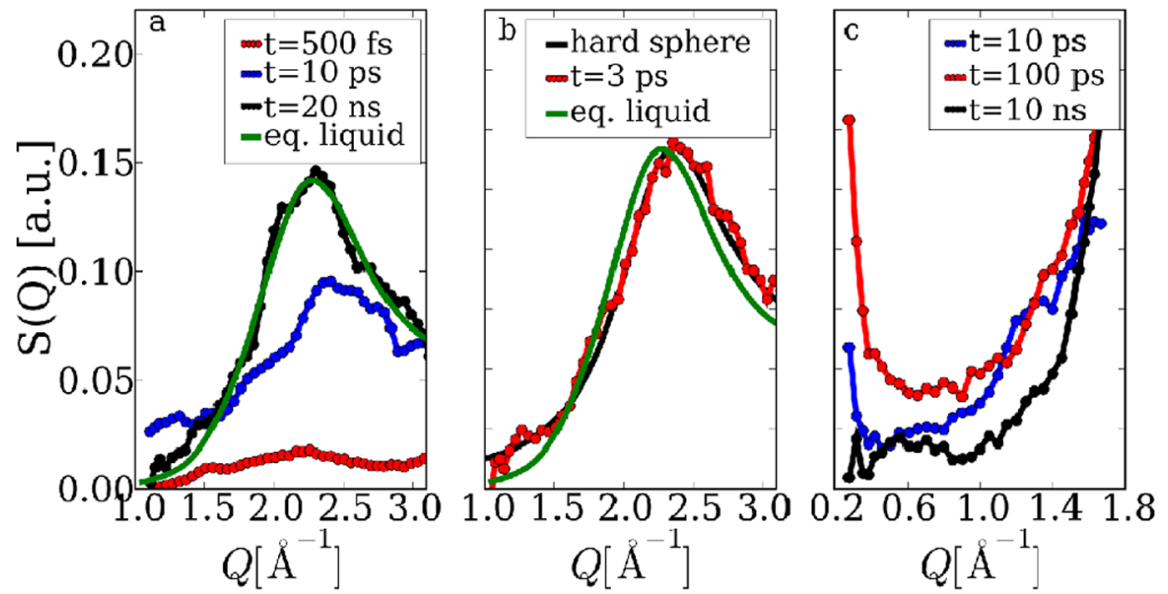

FIG. 2 (color online). (a) The structure factor at times $t=500 \mathrm{fs}, 10 \mathrm{ps}$, and $20 \mathrm{~ns}$, and compared to MD simulations [23] of the equilibrium liquid. (b) Comparison of the short time structure factor at $t=3 \mathrm{ps}$ to a hard-sphere model with parameters corresponding to the initial crystalline state. Also shown is a comparison to the equilibrium liquid. (c) Small-angle scattering measurements down to $0.2 \AA^{-1}$ at times $t=10 \mathrm{ps}, 100 \mathrm{ps}$, and $10 \mathrm{~ns}$.

tering under identical conditions. Near the peak of the liquid structure factor the increase in diffuse scattering occurs with a subpicosecond rise time, limited by our temporal resolution (700 fs, set by the crossing angle of the laser and $x$ rays on the sample), and coincident with the decrease in Bragg scattering. At small angles, the increase is significantly slower, occurring on $>10$ ps time scales. We interpret this behavior in the following way: In the limit as $Q$ approaches zero, the structure factor can be written as

$$
\left.\lim _{Q \rightarrow 0} S(Q)=1+\rho \int_{0}^{\infty}[g(r)-1)\right] d r=\frac{\left\langle N^{2}\right\rangle-\langle N\rangle^{2}}{\langle N\rangle}
$$

where $N$ is the number of atoms, $\rho$ is the density, and $g(r)$ is the pair correlation function [24]. The increase in scattering at low $Q$ thus indicates the development of longer length-scale density fluctuations in the liquid and the formation of a softer liquid than the equilibrium state $[25,26]$. The development of fluctuations will be limited by the speed of sound and the length-scale of the fluctuation; thus the low $Q$, longer length-scale dynamics will develop on slower time scales compared to the dynamics observed near the peak of the liquid structure factor, in agreement with Fig. 3. At longer time scales, the increase at low $Q$ is likely to be dominated by subsequent thermal and mechanical expansion of the liquid, occurring on hundreds of picosecond time scales. In contrast, during the first few ps, although the bonds have been broken, the volume is conserved. We note that, like the ice-water phase transition, crystalline InSb contracts upon melting [27]. A liquid at the initial crystalline density is thus generated during the first few ps, corresponding to a more open-packed structure than the equilibrium liquid. The short time scale compressibility is increased compared to the equilibrium state, and the system undergoes larger-amplitude density fluctu- ations, giving rise to the increase in scattering at small angles. We have quantitatively checked this hypothesis by calculating the liquid structure factor using a hard-sphere model [28], for which an analytical form exists with just one independent variable, the packing density of the liquid $\eta=\pi / 6 n \sigma^{3}$, where $n$ is the number density and $\sigma$ is the hard-sphere diameter. These calculations treat the initial optically induced liquid as a set of hard spheres with only short-range repulsive interactions, as would be expected at short times during which the covalent bonds are broken (this model does not accurately describe the equilibrium liquid). The packing density of the liquid is calculated from the initial parameters of the crystalline state, taking the nearest neighbor InSb distance, $2.81 \AA$ as the hard-sphere diameter, corresponding to $\eta=0.34$. Figure 2(b) shows a
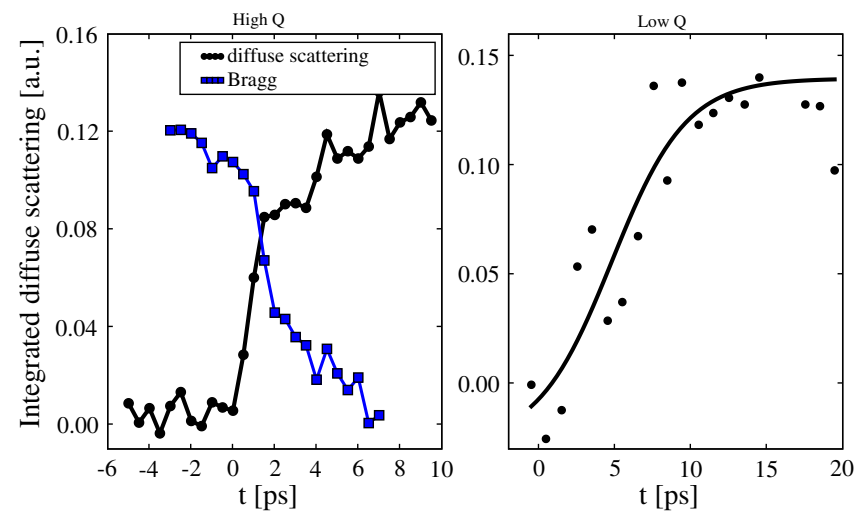

FIG. 3 (color online). Time scans recording the development of the diffuse scattering at both high and low $Q$. (left) Integrated diffuse scattering over $1.0-3.0 \AA^{-1}$. Also shown is the timedependence recorded on the (111) Bragg peak under identical excitation conditions. (right) Integrated diffuse scattering timedependence over a range $0.4-1.6 \AA^{-1}$. The solid line is a guide to the eye. 

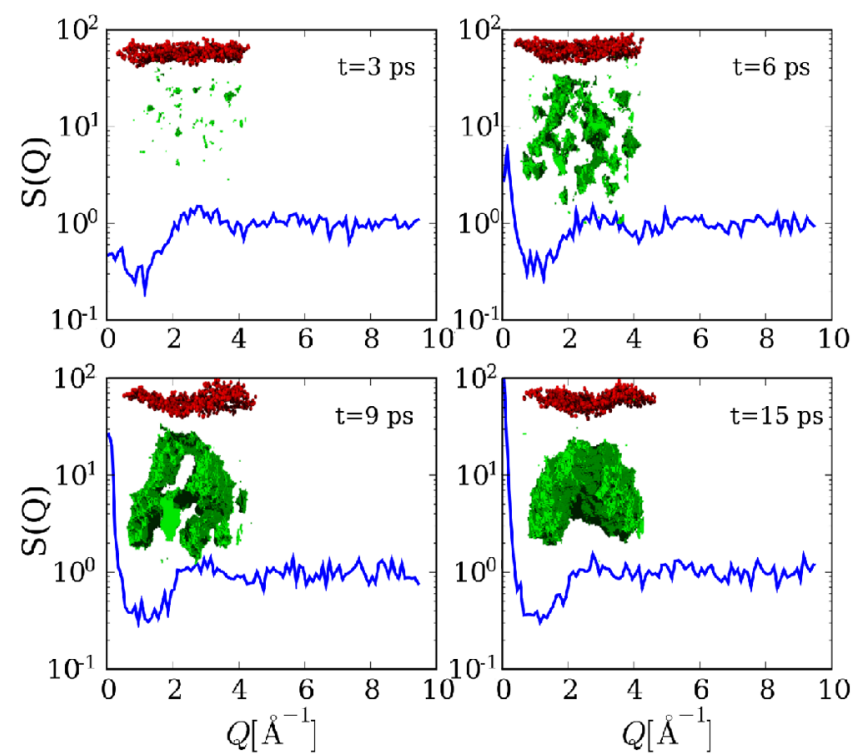

FIG. 4 (color online). Calculated structure factors for the case of Silicon just below the ablation threshold. (insets) Snapshots of the near-surface region at various times after excitation. The spontaneous development of voids (in green) below the surface (in red) is observed, leading to a quasidivergence in the low- $Q$ scattering.

comparison of the model calculation to the experimental data obtained at $t=3 \mathrm{ps}$, also compared to the equilibrium liquid structure factor. One observes that the hard-sphere model quantitatively reproduces the measured structure factor at short times, including the increase in scattering at low $Q\left(1-1.5 \AA^{-1}\right)$ that is observed compared to the equilibrium liquid. Thus, at short times, the measured transient liquid structure is qualitatively different from the equilibrium liquid, and is characterized by the structural properties of the initial crystalline state.

In order to probe the fluctuating nature of the photoinduced liquid state more closely, we extended our measurements to lower $Q$, probing down to $0.2 \AA^{-1}$. Figure 2(c) shows the measured diffuse scattering for $Q \in$ $\left(0.2-1 \AA^{-1}\right)$ at three different times, $t=10 \mathrm{ps}, t=$ $100 \mathrm{ps}$, and $t=10 \mathrm{~ns}$. At low $Q$ for $t=10 \mathrm{~ns}$, negligible scattering occurs. In contrast, one observes at $t=10 \mathrm{ps}$ and at $t=100 \mathrm{ps}$ a minimum in $S(Q, t)$ at nonzero $Q$ and a strong increase in the scattering near $Q=0$, indicative of the development of long range time-dependent correlations. For comparison, we have calculated the timedependent structure factor $S(Q, t)$ for a laser-excited semiconductor using previous molecular dynamics simulations for the case of silicon [22,29]. Si and InSb share similar phase diagrams, bonding, and structure so that the molecular dynamics (MD) simulations are expected to qualitatively describe our experimental results. The sample size was $10 \times 10 \times 30 \mathrm{~nm}$ and carrier and atom dynamics are described by Monte Carlo and MD techniques, respectively. The atoms follow Newtonian dynamics and interact via an empirical Stillinger-Weber potential which provides a satisfactory description of both the crystalline and liquid phases. The x-ray structure factor associated with calculated thermodynamic trajectories is shown in Fig. 4 during the first tens of picoseconds following excitation at a fluence near the ablation threshold. Calculated snapshots of the local structure near the surface are shown in Fig. 4 for each time. It is observed that on ps time scales, localized voids spontaneously form and then coalesce into a single nanoscale void or bubble, growing to a few nanometers in size, corresponding to the nucleation of the vapor phase and the first steps in the ablation of the material structure. The structure factor that follows from these MD simulations exhibits a transient divergence at small $Q$, extending to $\sim 0.5 \AA^{-1}$, similar to our experimental observations. These simulations thus provide a qualitative description of the fluctuating nature of the liquid at short times, in agreement with our experimental data.

The observed voids within the liquid represent the first steps in the nucleation of the vapor phase, a dynamical barrier crossing event in which a free energy barrier dictates a critical size for spontaneous growth of the new phase [1]. The experiments described above thus capture the nanoscale, ultrafast fluctuation dynamics that underlie superheated liquids, first order phase transitions in materials, and ablation physics, and provide the first direct measurements of the underlying atomic-scale dynamics. Future small-angle scattering measurements with spatially coherent femtosecond x-ray sources [13] will potentially enable measurement of the three-dimensional shape of the void structure and direct extraction of the free energy surface that determines the spontaneous nucleation dynamics in both bulk and nanoscale materials.

Portions of this research were supported by the U.S. Department of Energy, Office of Basic Energy Science through direct support for the SPPS, as well as individual investigators and SSRL, a national user facility operated by Stanford University. Additional support for the construction of SPPS was provided by Uppsala University and the Swedish Research Council. Portions of this work were supported by the Stanford PULSE Center and University of Michigan FOCUS Center. K. S. T. gratefully acknowledges financial support by the Deutsche Forschungsgemeinschaft (SFB 616 and SPP 1134). J.L., K.S.T., and M.N. acknowledge the support of the European Commission through the FEMTO, X-RAY FEL PUMPPROBE, and XPOSE projects. J.L. acknowledges support from Wallenberg Research Link and The Swedish Foundation for Strategic Research. K. J. G. and A. D. acknowledge support from the W. M. Keck Foundation.

*aaronl@stanford.edu

[1] D. Oxtoby, Acc. Chem. Res. 31, 91 (1998). 
[2] A. Pan, T. Rappl, D. Chandler, and N. Balsara, J. Phys. Chem. B 110, 3692 (2006).

[3] P. Debenedetti, Nature (London) 441, 168 (2006).

[4] D. Zahn, Phys. Rev. Lett. 93, 227801 (2004).

[5] H. Ihee et al., Science 291, 458 (2001).

[6] B. Siwick, J. Dwyer, R. Jordan, and R. Miller, Science 302, 1382 (2003).

[7] M. Harb et al., J. Phys. Chem. B 110, 25308 (2006).

[8] A. Zewail, Annu. Rev. Phys. Chem. 57, 65 (2006).

[9] H. Ihee et al., Science 309, 1223 (2005).

[10] A. Plech, V. Kotaidis, M. Lorenc, and J. Boneberg, Nature Phys. 2, 44 (2006).

[11] A. Plech et al., Phys. Rev. B 70, 195423 (2004).

[12] A. Lindenberg et al., J. Chem. Phys. 122, 204507 (2005).

[13] R. Service, Science 298, 1356 (2002).

[14] L. Bentson et al., Nucl. Instrum. Methods Phys. Res., Sect. A 507, 205 (2003).

[15] A. Lindenberg et al., Science 308, 392 (2005).

[16] A. Cavalieri et al., Phys. Rev. Lett. 94, 114801 (2005).

[17] D. Fritz et al., Science 315, 633 (2007).

[18] A. Rousse et al., Nature (London) 410, 65 (2001).
[19] K. Sokolowski-Tinten et al., Phys. Rev. Lett. 87, 225701 (2001).

[20] D. Perez and L. Lewis, Phys. Rev. Lett. 89, 255504 (2002).

[21] K. Sokolowski-Tinten et al., Phys. Rev. Lett. 81, 224 (1998).

[22] P. Lorazo, L. Lewis, and M. Meunier, Phys. Rev. B 73, 134108 (2006).

[23] T. Gu, X. Bian, J. Qin, and C. Xu, Phys. Rev. B 71, 104206 (2005).

[24] N. March and M. Tosi, Introduction to Liquid State Physics (World Scientific, Singapore, 2002).

[25] H. Bale, B. Dobbs, J. Lin, and P. Schmidt, Phys. Rev. Lett. 25, 1556 (1970).

[26] L. Bosio, J. Teixeira, and H. Stanley, Phys. Rev. Lett. 46, 597 (1981).

[27] X. Chen, Q. Wang, and K. Lu, J. Phys. Condens. Matter 11, 10335 (1999).

[28] N. Ashcroft and J. Lekner, Phys. Rev. 145, 83 (1966).

[29] P. Lorazo, L. Lewis, and M. Meunier, Phys. Rev. Lett. 91, 225502 (2003). 